\title{
Assessment of Styrene Oxide Neurotoxicity Using In Vitro Auditory Cortex Networks
}

\author{
Kamakshi V. Gopal, ${ }^{1,2}$ Calvin Wu, ${ }^{1,2,3}$ Ernest J. Moore, ${ }^{1,2}$ and Guenter W. Gross ${ }^{2,3}$ \\ ${ }^{1}$ Department of Speech and Hearing Sciences, University of North Texas, P.O. Box 305010, Denton, TX 76203-5010, USA \\ ${ }^{2}$ Center for Network Neuroscience, University of North Texas, P.O. Box 305010, Denton, TX 76203-5010, USA \\ ${ }^{3}$ Department of Biological Sciences, University of North Texas, P.O. Box 305010, Denton, TX 76203-5010, USA
}

Correspondence should be addressed to Kamakshi V. Gopal, gopal@unt.edu

Received 24 May 2011; Accepted 6 July 2011

Academic Editor: A. Bath

Copyright (C) 2011 Kamakshi V. Gopal et al. This is an open access article distributed under the Creative Commons Attribution License, which permits unrestricted use, distribution, and reproduction in any medium, provided the original work is properly cited.

Styrene oxide (SO) $\left(\mathrm{C}_{8} \mathrm{H}_{8} \mathrm{O}\right)$, the major metabolite of styrene $\left(\mathrm{C}_{6} \mathrm{H}_{5} \mathrm{CH}=\mathrm{CH}_{2}\right)$, is widely used in industrial applications. Styrene and SO are neurotoxic and cause damaging effects on the auditory system. However, little is known about their concentrationdependent electrophysiological and morphological effects. We used spontaneously active auditory cortex networks (ACNs) growing on microelectrode arrays (MEA) to characterize neurotoxic effects of SO. Acute application of 0.1 to $3.0 \mathrm{mM}$ SO showed concentration-dependent inhibition of spike activity with no noticeable morphological changes. The spike rate $\mathrm{IC}_{50}$ (concentration inducing $50 \%$ inhibition) was $511 \pm 60 \mu \mathrm{M}(n=10)$. Subchronic ( $5 \mathrm{hr})$ single applications of $0.5 \mathrm{mM} \mathrm{SO}$ also showed $50 \%$ activity reduction with no overt changes in morphology. The results imply that electrophysiological toxicity precedes cytotoxicity. Fivehour exposures to $2 \mathrm{mM}$ SO revealed neuronal death, irreversible activity loss, and pronounced glial swelling. Paradoxical "protection" by $40 \mu \mathrm{M}$ bicuculline suggests binding of SO to GABA receptors.

\section{Introduction}

Styrene is a colorless chemical solvent with an aromatic odor. It is extensively used in industries that manufacture polymers, plastics, and resins. Styrene enters the human body through several routes, especially the respiratory system. More than $80 \%$ of the inhaled styrene undergoes bioactivation to styrene oxide (SO) by cytochrome P450 monooxygenases [1]. SO is widely used also in industries as a diluent for epoxy resins and as a chemical intermediate in the manufacturing of cosmetics, agricultural chemicals, and surface coatings. A review of the literature indicates that there is a considerable amount of evidence that styrene and SO are neurotoxic although the precise mechanisms are unclear and quantitative data are lacking [2].

Exposure of low levels of styrene and its metabolites (including SO) may cause irritation of skin, eyes, and mucus membranes, but there is evidence that high doses can lead to neurological disorders [1]. The permissible styrene exposure limit set by the Occupational Safety and Health
Administration (OSHA) and the threshold limit value recommended by the American Conference of Industrial Hygienists is $50 \mathrm{ppm}$ (approximately $416 \mu \mathrm{M}$ ) for long-term exposure and $100 \mathrm{ppm}$ (approximately $850 \mu \mathrm{M}$ ) for shortterm exposure [3]. There has been no permissible exposure limits set for $\mathrm{SO}$ although $\mathrm{SO}$ is the most active metabolite formed from styrene.

Many of the adverse effects of styrene have been attributed to the accumulation of SO [4]. Otoneurologic tests on industrial workers with long-term styrene exposure at levels below $25 \mathrm{ppm}$ (approximately $200 \mu \mathrm{M}$ ) have revealed problems with the vestibular and auditory systems [5]. Other central nervous system problems induced by styrene exposure include vigilance, memory, vision, visuomotor performance, perceptual speed, and central auditory functions [5-7]. Styrene and SO are shown to impede the functioning of various neurotransmitters in the brain including dopamine and serotonin although uncertainties exist about the exact nature of the hindrance caused by these compounds $[3,8,9]$. The cytotoxicity from styrene and SO exposure is 
thought to be similar to oxidative stress-induced conditions caused by oxidizing protein thiols [4]. Primary cerebellar granule neurons and human neuroblastoma cells exposed to SO $(0.3$ to $1 \mathrm{mM})$ induced apoptosis, which can be triggered by oxidative stress $[1,10-12]$. There is also evidence that exposure of primary striatal neurons to $\mathrm{SO}$ induces synaptic impairments [2], which might be the reflection of morphological alteration of the neuronal cytoskeleton. Furthermore, these data supported the hypothesis of reactive oxygen species initiating the events of SO cytotoxicity. $\mathrm{SO}$ is a proven animal carcinogen and is classified as a possible human carcinogen (group 2B) by the International Agency for Research on Cancer [13].

Johnson et al., 2006, reviewed nine studies that examined the relationship between occupational exposure to styrene and hearing loss [14]. They found that in seven of the nine studies, there was an association between styrene exposure and hearing loss. Occupational exposure to styrene levels of 40-50 ppm for more than 10 years showed elevated hearing thresholds at frequencies up to $1500 \mathrm{~Hz}$ [15]. However, at lower concentrations of styrene (below $20 \mathrm{ppm}$ ), no association between exposure and hearing deficit was found. Chen et al., 2008, reported styrene-induced cochlear injury prior to functional loss in an animal model [16]. Exposure to styrene in the presence of industrial noise is shown to have a synergistic effect on the hearing loss incurred by animals and humans [17-19]. Styrene exposure in combination with noise levels within recommended limits has an effect on the auditory system [20]. Chen and Henderson, 2009, have suggested that individual exposure to noise or styrene may cause stress, temporary alteration, or nonlethal injury to cochlear hair cells, but the combination exposure of noise and styrene strengthens the stress on the hair cells, leading to cell death [21].

Neurophysiologic testing of brain dysfunction demonstrates reaction time deficiencies in people exposed to styrene [22]. Studies have also shown that styrene has adverse effects on the performance of the central auditory system, including temporal processing skills [14, 23-25]. The European Directive (EU 2003) has specified that risk assessment should include interactions of noise and work-related ototoxic substances such as styrene [26]. NIOSH has recommended establishment of exposure limits for ototoxic chemicals in the presence and absence of noise [27]. Styrene ranks at the top of the list along with toluene as a potentially ototoxic solvent that is widely used in industrial settings [28]. There is, however, a limited understanding of the effects of styrene or its major metabolite $\mathrm{SO}$ on the central auditory system.

This study was undertaken to assess the toxicity of SO using an in vitro model of auditory cortex networks (ACNs) growing on multielectrode arrays (MEAs). The objective was to characterize electrophysiological (functional) toxicity and cellular toxicity for acute and subchronic SO exposures and determine if functional toxicity preceded cellular toxicity. Acute neurotoxicity was assessed by serial additions of the SO to mature cultures (21 div or older) that were spontaneously active. The criterion time point selected was 30 minutes at each concentration. For subchronic neurotoxicity assessment, mature cultures that were spontaneously active were exposed to a single concentration of SO for five hours. The concentration-response relationship of $\mathrm{SO}$ was compared to exposure levels of styrene seen in industrial settings, since no such levels are currently available for SO exposure.

\section{Materials and Methods}

2.1. Cell Culture. The cell culture techniques using ACNs have been published earlier [29-31]. This study was approved by the University of North Texas Institutional Animal Care and Use Committee. Briefly, auditory cortices were dissected from E16-E17 Balb-C/ICR mouse embryos, and were subjected to the standard culturing procedures. The dissociated neurons were seeded at a density of approximately 10,000 cells $/ \mathrm{mm}^{2}$ on MEAs with substrate-integrated microelectrodes [29]. The networks were maintained in the incubators at $37^{\circ} \mathrm{C}$ in a $10 \% \mathrm{CO}_{2}$ atmosphere and fed twice a week using half-medium changes of Dulbecco's modified minimum essential medium (DMEM) supplemented with $5 \%$ horse serum. On the day of the experiment, the original medium was completely replaced with serum-free DMEM (stock medium).

2.2. Microelectrode Arrays (MEA). Previous publications have described in detail the procedures adopted in the inhouse MEA fabrication [32-34]. Briefly, the MEAs were photoetched from commercially available indium-tin oxide (ITO) plates (Applied Films Corp., Boulder, Colo, USA) to generate $5 \mathrm{~cm}^{2}$ and $1 \mathrm{~mm}$ thick plates with 32 amplifier contact strips on either side. The contact strips terminated in the center of the plate in a $0.8 \mathrm{~mm} \times 0.8 \mathrm{~mm}$ recording matrix consisting of 64 recording sites (electrodes). The electrode terminals were either arranged in 4 rows and 16 columns or in 8 rows and 8 columns and conductors measured $1000 \AA$ in thickness and $8 \mu \mathrm{M}$ in width. The processed ITO plates were spin insulated with methyltrimethoxysilane resin and deinsulated at the conductor tips with single laser shots. The exposed metal sites were then gold plated to lower the electrode impedance at $1 \mathrm{kHz}$ to approximately $0.8 \mathrm{mOhms}$.

2.3. Electrophysiological Recording. The MEA recording techniques used in the study have also been described in detail in previous publications $[29,32]$. The matrix region was treated with poly-D-lysine and laminin to support adhesion of dissociated cells. For electrophysiologic recording, only ACNs that were at least three weeks old in vitro were used. By three weeks after seeding, the neurons develop shallow, three-dimensional networks with neuronal cell bodies generally situated on top, and neural processes situated above and below the glial carpet [35]. Moreover, neuronal networks grown in this manner remain spontaneously active and pharmacologically responsive for more than 6 months [32].

On the day of the experiment, the cultures were maintained at $37 \pm 0.5^{\circ} \mathrm{C}$ on an inverted microscope stage in a special recording chamber [32]. The recording chamber allows for network maintenance in a constant bath of 1 to $2 \mathrm{~mL}$ medium and is well suited for rapid medium changes and short-term (24 hours or less) pharmacological studies. The $\mathrm{pH}$ was stabilized at 7.4 by passing a stream of 
humidified $10 \% \mathrm{CO}_{2}$ in air through a cap fitted with a heated ITO window to prevent condensation, which permitted microscopic observations. Osmolarity was maintained at 300 to $320 \mathrm{mOsm}$ by adding water at a rate of $65 \mu \mathrm{L} / \mathrm{hr}$ via a syringe pump. Neuronal activity was recorded with a twostage, 64 channel amplification and signal processing system (Plexon Inc., Dallas) with a total system gain set at $10 \mathrm{k}$. Channels were assigned to 64 digital signal processors with a $40 \mathrm{kHz}$ sampling rate. Waveshapes representing single active units were discriminated via template matching. Up to four different templates per physical channel could be discriminated in real time and assigned to separate logical channels. To follow the behavior of the network before, during, and after test compound application, data were displayed as mean network activity per minute. Channels with best signal-to-noise ratios were selected for further monitoring.

2.4. Experimental Protocol. Following the assembly on the recording chamber, the ACNs were allowed to stabilize in fresh medium consisting of stock DMEM without serum. The spontaneous activity was generally recorded for a minimum of 30 minutes and was termed "reference activity". Following this period, addition of SO (Sigma-Aldrich, St Louis, Mo, USA) was undertaken, while the ongoing activity was continuously recorded. SO stock solution was prepared in dimethyl sulfoxide (DMSO), since SO is only slightly soluble in water. The maximum DMSO volume in the bath did not exceed $4 \%$ of the total bath volume. Control studies were done with DMSO alone to account for its independent effects on the ACNs. For acute experiments, SO dissolved in DMSO was added to the bath with a micropipette to achieve final concentrations ranging from 100 to $3000 \mu \mathrm{M}$, mimicking the styrene levels of $12 \mathrm{ppm}$ to $360 \mathrm{ppm}$ in occupational exposures in vivo [2]. The network activity was continuously monitored for about 30 minutes prior to the addition of the next dosage to allow for network stabilization. For subchronic experiments, ACNs were exposed to a onetime application of 0.5 or $2 \mathrm{mM} \mathrm{SO}$, and the activity was monitored continuously for five hours before a complete wash (replacement of the medium with fresh stock DMEM) was undertaken. The cells were monitored optically for morphological changes during acute and subchronic experiments. Cell stress was determined by observations of vesiculation, swelling, obscuration of nucleus, and phase brightness. In a subset of experiments, bicuculline $(40 \mu \mathrm{M})$ was added prior to addition of SO.

2.5. Data Analysis. To establish SO-induced changes in the ACNs, mean spike rates were obtained for each concentration level. To quantify the changes induced by SO, the reference activity was compared to network activity at each concentration of SO, and a normalized percent change was obtained. Independent samples $t$-test was used to identify if the reduction in spike activity seen in ACNs exposed to $\mathrm{SO}$ was significantly more than the reduction of spike activity in ACNs exposed to DMSO only. A criterion alpha level of 0.05 was adopted for the comparison. All computations were conducted using the Statistical Package for the Social

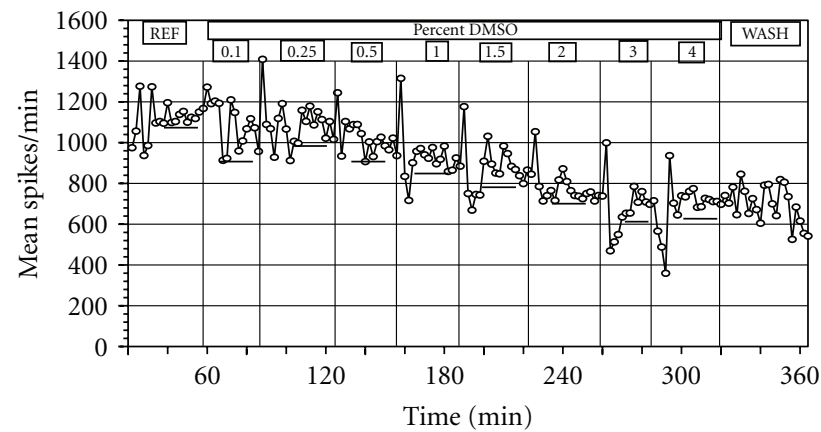

(a)
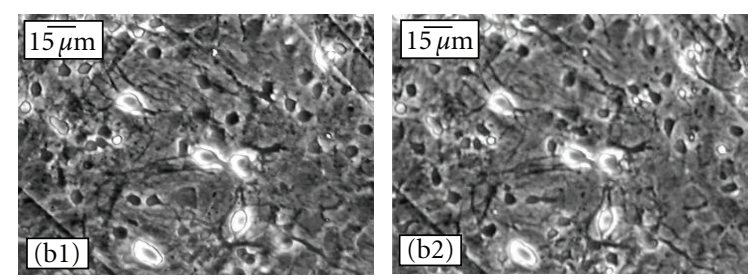

(b)

FIgURE 1: Effects of DMSO on ACN spontaneous activity and morphology. (a) A gradual decrease in mean spikes per min from 26 units. The sudden augmentation of activity seen during the addition of the test compound is due to mixing of the compound in the bath medium. Horizontal bars represent quasistable states used or quantification of activity changes. (b) Neurons and glia in reference medium (b1) and after $200 \mathrm{~min}$ in $4 \%$ DMSO (b2). No overt changes in neuronal morphology (phase bright cells) can be identified.

Sciences (SPSS) software. It is important to note that single cells are not reliable indicators of pharmacological responses. Population responses are more fault tolerant and provide representative dose-response functions [36].

\section{Results}

The ACNs used in this study ranged in age from 21 to 42 days in vitro, with a mean age of $31 \pm 6.6$ days. This study evaluated acute (30 minute exposure) and semichronic effects (five hour exposure) of SO on electrophysiological activity and morphological aspects of ACNs. Independent effects of DMSO were first evaluated for reference purposes followed by investigations of the effects of $\mathrm{SO}$ dissolved in DMSO. Further, the effects of SO on ACNs exposed to $40 \mu \mathrm{M}$ bicuculline (a competitive antagonist of $\mathrm{GABA}_{\mathrm{A}}$ receptors) were also examined.

3.1. Acute Effects of DMSO on ACNs. Sequential application of DMSO induced a concentration-dependent inhibition of network spike activity. Figure 1(a) depicts the responses from an ACN that was subjected to DMSO at concentrations ranging from $0.01 \%$ to $4 \%$ followed by a complete medium change. The average spike rate decreased as a function of DMSO concentration. To identify morphological changes, neurons and neuronal processes in the matrix area were monitored throughout the experiment. No significant morphological changes were identified between reference (no DMSO) and 4\% DMSO (Figure 1(b)). 


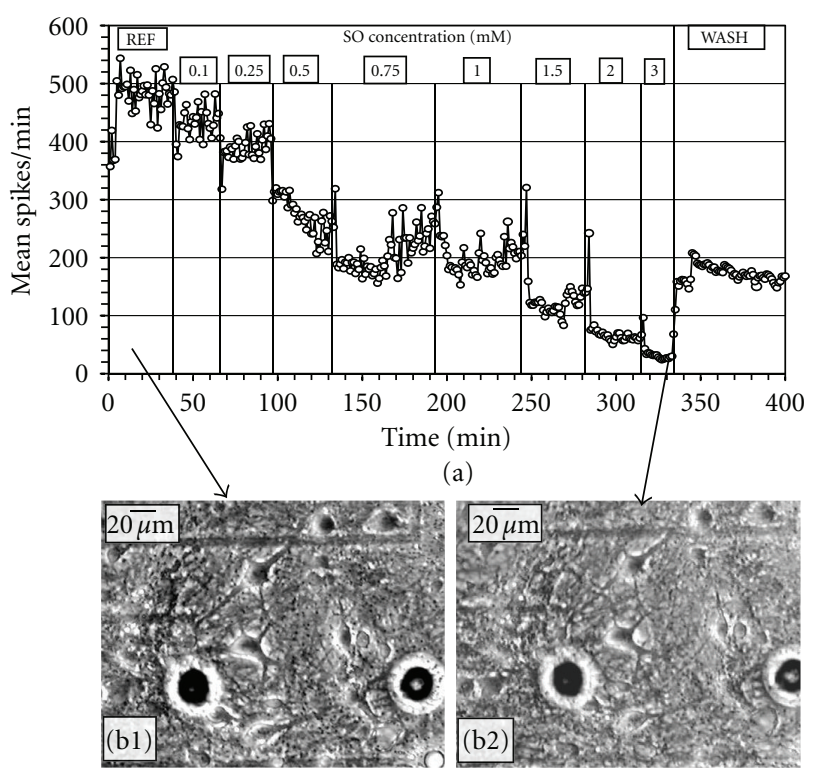

(b)

Figure 2: Acute effects of SO on ACN spontaneous activity and morphology. (a) A stepwise dose-dependent inhibition of mean spike rate per min (15 units). The activity was partially reversible with a single wash. (b) Neurons and glia in reference medium (b1) and after $20 \mathrm{~min}$ in $3.0 \mathrm{mM}$ SO (b2). No overt changes in morphology can be identified. The round black circles seen in the figures are gold-plated electrodes.

TABLE 1: Percent average spike rate reduction with acute application of DMSO and SO (maximum DMSO $=3 \%$ ).

\begin{tabular}{|c|c|c|c|c|}
\hline \multicolumn{2}{|c|}{ Concentrations } & \multicolumn{2}{|c|}{ Percent activity decrease } & \multirow{2}{*}{$\begin{array}{c}\text { Percent difference } \\
\text { (SO effect) }\end{array}$} \\
\hline $\begin{array}{l}\mathrm{SO} \\
(\mathrm{mM})\end{array}$ & $\begin{array}{c}\text { DMSO } \\
(\%)\end{array}$ & $\begin{array}{c}\text { DMSO only } \\
\quad(n=3)\end{array}$ & $\begin{array}{l}\text { SO in DMSO } \\
\quad(n=10)\end{array}$ & \\
\hline 0.1 & 0.1 & $10.8 \pm 5.9$ & $17.6 \pm 3.3$ & 6.8 \\
\hline 0.25 & 0.25 & $13.1 \pm 8$ & $32.7 \pm 6.6$ & 19.6 \\
\hline 0.5 & 0.5 & $17.1 \pm 2.7$ & $45.9 \pm 4.1$ & 28.8 \\
\hline 1.0 & 1.0 & $25.9 \pm 6.4$ & $59.5 \pm 6.2$ & 33.6 \\
\hline 2.0 & 2.0 & $36.5 \pm 7$ & $76.2 \pm 7.6$ & 39.6 \\
\hline 3.0 & 3.0 & $39.8 \pm 8.1$ & $81.1 \pm 7.7$ & 41.3 \\
\hline
\end{tabular}

3.2. Acute Effects of SO. With cumulative application of $\mathrm{SO}$ (dissolved in DMSO), a pronounced concentration-dependent spike rate inhibition was noticed. Figure 2(a) shows a typical $\mathrm{ACN}$ response to $\mathrm{SO}$ addition: gradual stepwise reduction in average spikes as a function of concentration. At $3.0 \mathrm{mM}$ SO, more than $90 \%$ of the spiking activity was lost. When the culture was subjected to a complete wash, there was partial (38\%) recovery of the activity. The spike rate $\mathrm{IC}_{50}$ (concentration inducing 50\% inhibition) occurred at approximately $500 \mu \mathrm{M}$. Morphological analysis of the neurons monitored throughout the experiment showed no noticeable changes in cell morphology (Figure 2(b)).

Table 1 shows the average reduction of mean spike activity in ACNs exposed to DMSO alone ( $N=3$ experiments), and to SO dissolved in DMSO $(N=10$ experiments $)$.

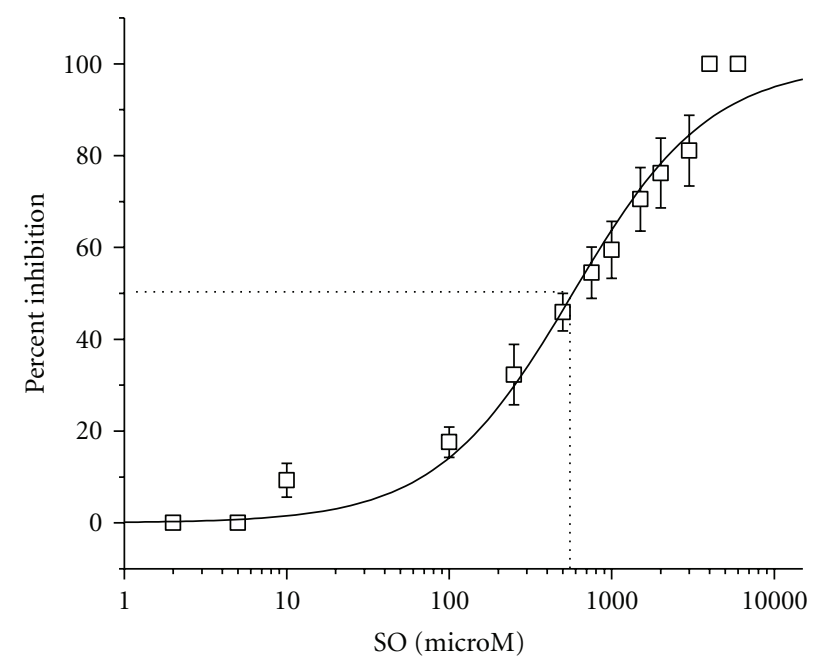

Figure 3: Concentration-response curve for SO. Percent inhibition of network spike rate per concentration of SO was calculated for each network using the specific reference activity of that network and averaged. The spike rate $\mathrm{IC}_{50}$ mean $\pm \mathrm{SE}$ is $511 \pm 60.1 \mu \mathrm{M}$ SO.

A significantly greater reduction in activity was obtained for ACNs exposed to SO compared to DMSO alone. This difference was significant at the 0.05 level $(t$-test, $P=0.03$ ). With a single wash following $3 \mathrm{mM}$ SO application, the activity recovered to only to $28.9 \pm 8.9 \%$ of the original reference level. This shows that the inhibitory effects of SO remain even after a full medium change.

3.3. Concentration-Response Characteristics. The concentration-response curve obtained from 10 ACNs (total of 203 neurons) exposed to acute sequential application of $\mathrm{SO}$ is shown in Figure 3. ACNs displayed inhibitory monotonic responses with sequential addition of $\mathrm{SO}$. The $\mathrm{IC}_{50}$ value, which represents the mean concentration at which spike rates were inhibited $50 \%$ of their original level, was $511 \pm 60.1 \mu \mathrm{M}$.

3.4. Subchronic (Five-Hour Exposure) Functional and Cellular Effects of SO on ACNs. Since the permissible exposure limit set by OSHA for styrene is $50 \mathrm{ppm}$ (approximately $416 \mu \mathrm{M}$ ), which is close to our $\mathrm{IC}_{50}$ concentration of $511 \mu \mathrm{M}$, we investigated the effects of a single dose of $\mathrm{SO}$ application at a concentration of $0.5 \mathrm{mM}$ on ACNs for a period of five hours (to represent a subchronic condition). Additionally, for comparison purposes, we evaluated the effects of a one-time $2.0 \mathrm{mM}$ sub-chronic exposure. The changes were monitored electrophysiologically as well as morphologically for five hours.

Figure 4(a) depicts the effects of a one-time (nonsequential) application of $0.5 \mathrm{mM} \mathrm{SO}$ on an ACN resulting in an immediate cessation of activity. A rapid spontaneous recovery followed, leading to an eventual activity stabilization at approximately $40 \%$ of the original reference activity. A complete wash did not facilitate any recovery in this experiment. Morphologically, the neurons appeared to be normal and healthy throughout the duration of the experiment (Figure 4(b)). 


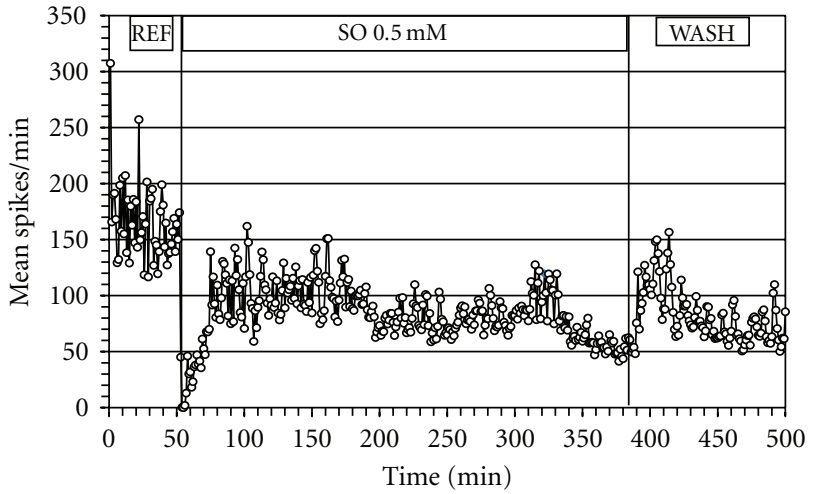

(a)

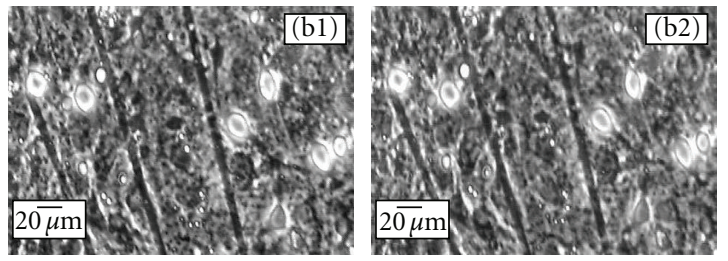

(b)
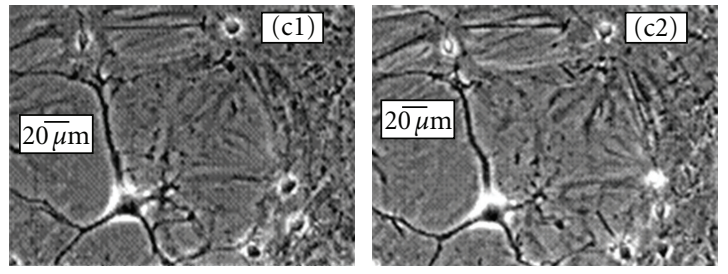

(c)

FIgURe 4: Subchronic effects of $0.5 \mathrm{mM}$ SO (in 0.5\% DMSO) on ACN spontaneous activity and morphology. (a) Mean spike rate per min from 33 units show initial cessation of activity followed by recovery of activity that stabilized to about $40 \%$ of the original level at the end of five hours. A complete wash did not lead to any further recovery. (b) and (c) Neurons and glia in reference medium (1) and after 5 hrs in $0.5 \mathrm{mM}$ SO (2). Only minor changes in morphology can be identified.

Figure 5(a) shows the effect of a one-time (nonsequential) application of $2.0 \mathrm{mM} \mathrm{SO}$ on an ACN. The single application of $\mathrm{SO}$ caused a more drastic effect compared to the sequential application of SO (as seen in Figure 2(a)). The activity initially ceased, followed by a weak recovery period prior to a complete loss of activity. This was accompanied by a partial loss of neurons and massive glial swelling as seen in Figure 5(b). The glial swelling was so extensive that optical identification of neurons was difficult.

The average spike activity in 3 ACNs exposed to a onetime $0.5 \mathrm{mM}$ for five hours showed reduction to $50.7 \pm 8.4 \%$ of its original level. Recovery of activity following a complete medium change was less than $50 \%$ of the original reference level. Data from $3 \mathrm{ACNs}$ exposed to $2.0 \mathrm{mM}$ SO showed reduction to $92.5 \pm 5.8 \%$ of its original level within five hours, with no recovery following a complete medium change.

Data obtained so far show a rapid response to the addition of $\mathrm{SO}$ that is most obvious at the higher concentrations. The recovery process showed a slow rise of activity of approximately $7 \%$ per $10 \mathrm{~min}$ and a rapid rise that doubles activity in $10 \mathrm{~min}$ (Figure $6(\mathrm{a}), 1 \mathrm{mM}$ ), suggesting two

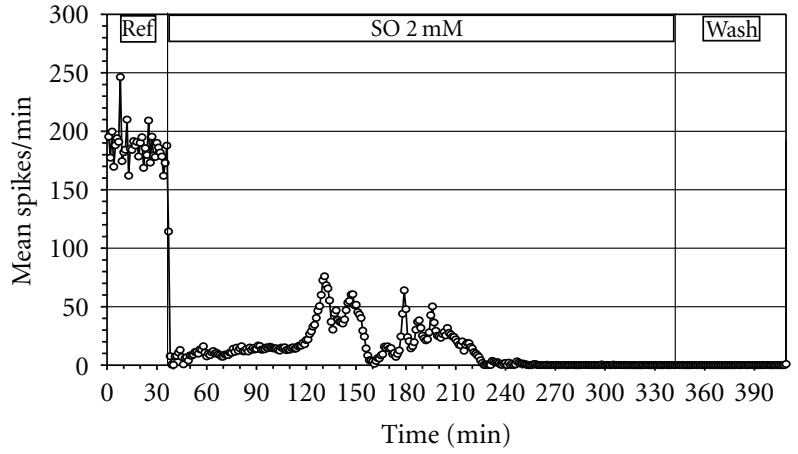

(a)
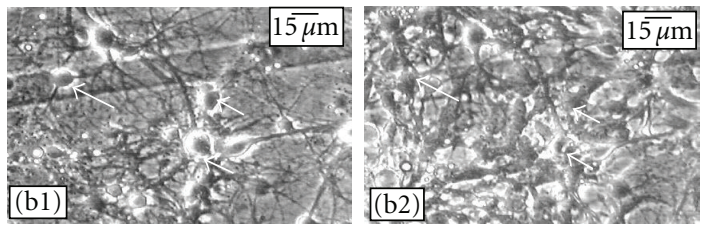

(b)
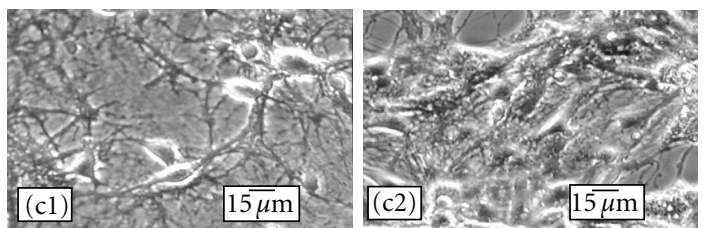

(c)

Figure 5: Subchronic effects of $2.0 \mathrm{mM}$ SO (in 2\% DMSO) on ACN spontaneous activity and morphology. (a) Mean spike rate per min from 24 units show initial cessation of activity and slow recovery of activity lasting for about an hour that was abolished in $3.5 \mathrm{hrs}$. A complete wash did not lead to any recovery of activity. (b) and (c) Neurons and glia in reference medium (1) and after $5 \mathrm{hrs}$ in $2.0 \mathrm{mM}$ SO (2). Although some neuronal death is present (arrows) the most obvious response is a massive glial swelling. (b) and (c) Are different networks. $\mathrm{c} 2$ is not the same area as c1. Arrows in (b) point to reference neurons for orientation.

possible mechanisms. At $2 \mathrm{mM}$, almost all activity was lost with no recovery. Under $40 \mu \mathrm{M}$ bicuculline (Figure 6(b)) three different slopes appeared, suggesting three possible different dynamic mechanisms. Further, activity was not lost at $2 \mathrm{mM}$ over an observation period of $350 \mathrm{~min}$. SO titration in the presence of bicuculline (Figure $7(\mathrm{a})$ ) showed retention of about $50 \%$ activity at $2.0 \mathrm{mM}$ and even $30 \%$ of reference at $4 \mathrm{mM}$ (data not shown). The concentration-response curve (Figure 7(b)) revealed a large shift to higher concentrations with and $\mathrm{IC}_{50}$ change from approximately 500 to $1400 \mu \mathrm{M}$ ( $n=10 \mathrm{ACNs}$ ). The slope of this curve is close to the standard curve (a Hill slope of 1), implying competitive antagonism at the GABA receptor.

\section{Discussion}

Styrene and its major metabolite $\mathrm{SO}$ have been used in industries for more than 100 years. However, their effect on the auditory system has only recently come to the attention of investigators. A strong link between exposure to 


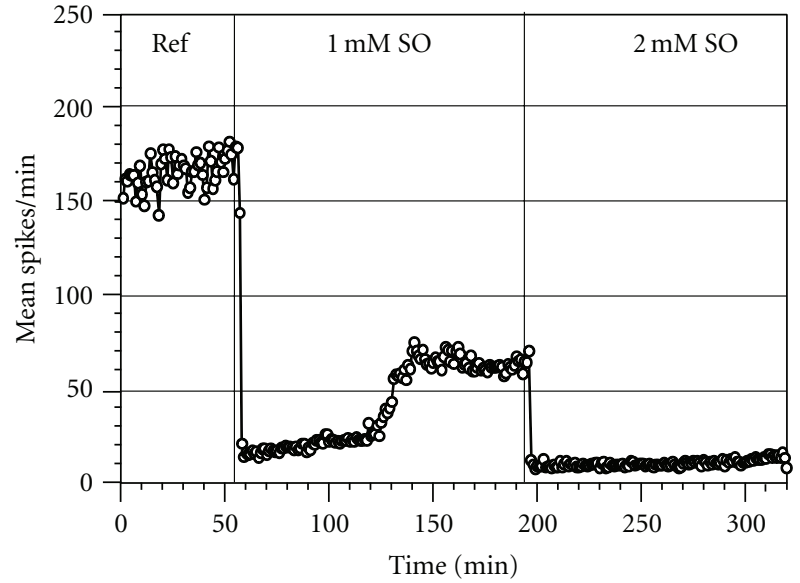

(a)

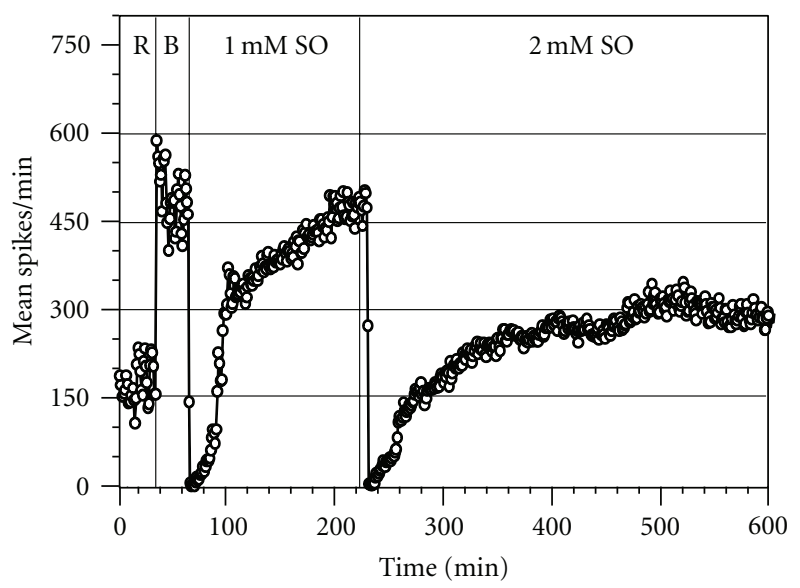

(b)

FIGURE 6: Spontaneous recovery from SO application (1.0 and $2.0 \mathrm{mM})$ and influence of $40 \mu \mathrm{M}$ bicuculline. (a) The network shows a $35 \%$ recovery at $60 \mathrm{~min}$ after $1.0 \mathrm{mM}$ SO application. Note the rapid loss of activity (1-2 min) implying interactions with membrane surface receptors. A $2.0 \mathrm{mM}$ application of SO reduced activity by more than $90 \%$, with no recovery. (b) In the presence of bicuculline, the network shows almost complete recovery to the bicuculline reference with $1.0 \mathrm{mM}$ SO. A $2.0 \mathrm{mM}$ SO application stops all activity followed by a spontaneous recovery to more than $50 \%$ of reference. $\mathrm{R}=$ Reference; $\mathrm{B}=$ Bicuculline.

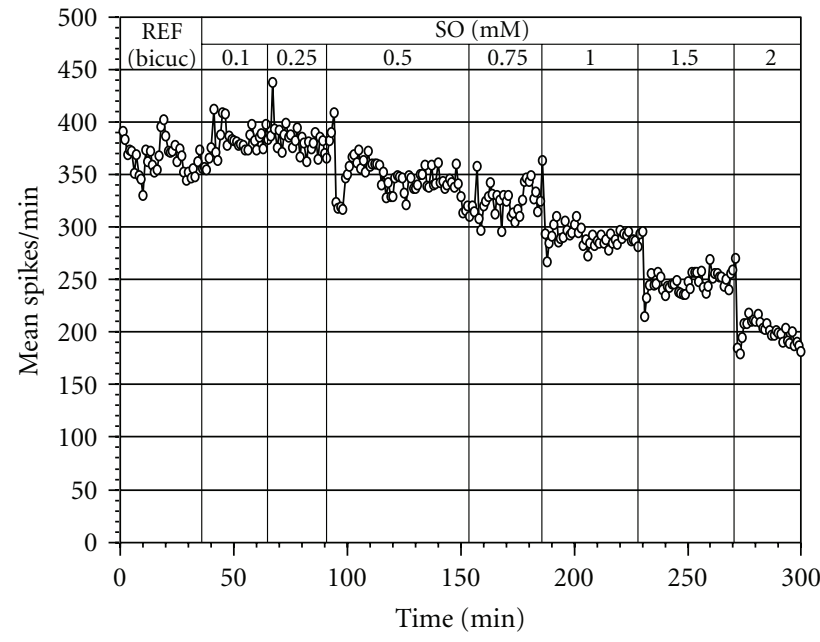

(a)

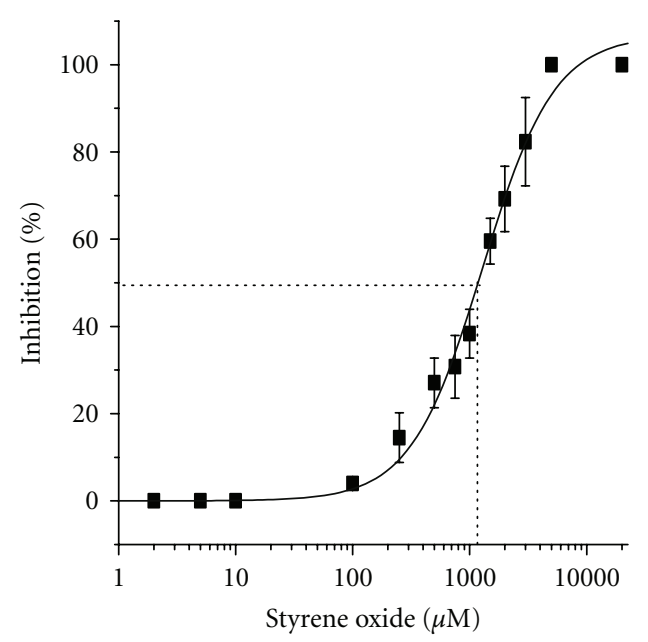

(b)

FIGURE 7: (a) Influence of bicuculline on network response to styrene oxide titration. Reference activity represents pretreatment with $40 \mu \mathrm{M}$ bicuculline. The concentration-dependent inhibition of spike activity is significantly less in the presence of bicuculline. (b) Concentration response curve from SO titration experiments under bicuculline $(n=10)$. The $\mathrm{IC}_{50}$ shifted from $511 \mu \mathrm{M}$ (Figure 3 ) to $1405 \mu \mathrm{M}$. The slope is close to the standard curve with a slope of 1, implying competitive antagonism at the GABA receptors.

styrene and "sensorineural" hearing loss, mainly retrocochlear and central problems in industrial workers, has been shown, thus substantiating the notion that styrene is neurotoxic and affects the central auditory nervous system $[14,15,23-25]$. Using multichannel electrophysiological recordings of spontaneously active ACNs growing on MEAs, we tracked the temporal evolution of the effects of $\mathrm{SO}$ together with concomitant morphological changes.

Toxicity of SO was more readily seen using electrophysiological recordings rather than morphological observations. Exposure of ACNs to DMSO induced reduction in electrophysiological activity with no overt signs of morphological damage. The reduction in activity, however, was significantly higher when ACNs were exposed to SO dissolved in DMSO. Addition of SO exhibited steeper concentration-dependent inhibition of network spike activity. Although the $\mathrm{IC}_{50}$ value was close to $0.5 \mathrm{mM}$, morphological analysis of acute exposure to SO up to $3.0 \mathrm{mM}$ concentration did not show any overt signs of morphological damage. This substantiates the fact that electrophysiological recordings provide a more sensitive index of toxic effects than morphological observations. Exposure of $0.5 \mathrm{mM}$ SO subchronically (for five hours) showed approximately a 50\% reduction in activity, with no overt signs of cellular damage. However, 
a complete medium change did not lead to a sizeable recovery, suggesting irreversible electrophysiological effects of SO. Subchronic exposure of $2 \mathrm{mM}$ SO brought about rapid ( $2 \mathrm{~min}$ ) cessation of activity and partial neuronal death within five hours. However, it must be noted that when ACNs were exposed acutely to $2.0 \mathrm{mM} \mathrm{SO}$ in a sequential (cumulative) manner (Figure 2(a)), the functional effects were not as drastic and the cellular effects were not readily observed compared to the responses seen with a one-time noncumulative application of $2.0 \mathrm{mM}$ SO. The functional changes observed in the concentration-dependent responses cannot be directly attributed to overt neuronal damage, because the morphology of the neurons was not affected at concentration levels that brought about electrophysiological changes.

An unexpected finding was the extensive swelling of glia (Figure 5) after a five-hour $2.0 \mathrm{mM}$ exposure. The relatively flat glial control carpet erupts into a highly convoluted structure. Quantification of this phenomenon is difficult, as the glia cannot be easily identified in the control state without special staining. However, the swelling represent a major cellular change that is very likely to disrupt cell-electrode coupling. Hence, the loss of activity is at least partially due to this major structural disruption. Although some neuronal death can be morphologically verified, the changes in the glial carpet obscure many details and neuronal death quantification must be performed with staining in a future study. Closer examination also indicates that not all nonneuronal cell types participate. It is possible (but not yet proven) that only astrocytes respond to SO with extensive swelling. It is important to consider that such glial reactions in an animal can lead to a great variety of effects on local blood supply and on electrical activity, culminating eventually in neuronal death.

The almost immediate network response to higher concentrations of $\mathrm{SO}$, the spontaneous recovery, and the "protection" by $40 \mu \mathrm{M}$ bicuculline (Figure 6(b)) are also surprising but highly repeatable observations. The rapid loss of network activity implies SO interactions with a plasma membrane receptor at synapses. Such a response can be generated by an increase in network inhibition and also by a decrease in network excitation A subsequent spontaneous recovery implies either changes in the pertinent receptor population and/or a compartmentalization of SO, presumably into membranes. The recovery of spontaneous activity following high concentration of SO seemed to include a slow rise of activity followed by a rapid rise (Figure 6(a), $1 \mathrm{mM}$ ). At $2.0 \mathrm{mM}$, most activity was lost with no recovery even after a maximum observed period of 10 hours (data not shown). In the presence of $40 \mu \mathrm{M}$ bicuculline, three different slopes appeared, suggesting three different dynamic mechanisms (Figure 6(b)), Further, the activity shows retention of about $50 \%$ activity at $2.0 \mathrm{mM}$ and even $30 \%$ of reference at $4 \mathrm{mM}$ (data not shown). Additionally, the concentration-response curve depicted in Figure 7(b) revealed a major shift of the $\mathrm{IC}_{50}$ value in $\mathrm{ACN}$ exposed to bicuculline. The slope of this curve implies competitive antagonism at the GABA receptors. It appears that we are observing the combined effects of (a) SO agonistic activity at GABA receptors, (b) changes in cell/electrode coupling due to glial swelling, and (c) neuronal cell death (at high concentrations). In light of reported reactive oxygen species production by SO [4], it is reasonable to suggest that impaired mitochondrial function and associated impaired ionic gradients lead to osmotic swelling. Such a mechanism should affect all cells but not necessarily at the same rates.

This study has demonstrated that ACNs exposed to SO show concentration-dependent electrophysiological toxicity even at limits set by OSHA for long- and short-term styrene exposure. Although no overt cellular deficits were noticed for acute or subchronic exposures of $0.5 \mathrm{mM} \mathrm{SO}$, electrophysiological deficits leading to as much as a $50 \%$ reduction of activity and incomplete recovery after medium changes signifies the depressant and irreversible effects of SO. However, it is not clear whether impaired reversibility is due to a loss of neurons or to a change in cell-electrode coupling. Styrene exposure, even at concentrations below the OSHA limit, can possibly lead to subtle effects on the auditory system leading to hearing loss and central auditory processing difficulties $[5,37]$. Hence, it is recommended that auditory evaluation and monitoring of styrene-exposed individuals should include central auditory test measures. To our knowledge, the swelling of glia has not been reported previously and must be considered a key player in the observed neurological disturbances in humans. Certainly, pressure on neuronal cell bodies or even axons can lead to abnormal evoked activity that could lead to hearing loss and possible associated symptoms such as tinnitus. The glial swelling and the assumed compartmentalization of SO, leading to partial spontaneous recovery, are surprising observations that require further study.

\section{Acknowledgments}

This study was supported by a grant awarded to K. V. Gopal by the Faculty Research Grants Program at the University of North Texas and the Charles Bowen Memorial Endowment to the CNNS. The authors thank CNNS staff members Sarah Valliere and Agustin deLaRosa for cell culture support and Ahmet Ors for the fabrication of microelectrode arrays.

\section{References}

[1] E. Dare, R. Tofighi, M. V. Vettori et al., "Styrene 7,8-oxide induces caspase activation and regular DNA fragmentation in neuronal cells," Brain Research, vol. 933, no. 1, pp. 12-22, 2002.

[2] P. Corsi, A. D’Aprile, B. Nico, G. L. Costa, and G. Assennato, "Synaptic contacts impaired by styrene-7,8-oxide toxicity," Toxicology and Applied Pharmacology, vol. 224, no. 1, pp. 4959, 2007.

[3] F. Gagnaire, M. Chalansonnet, N. Carabin, and J. C. Micillino, "Effects of subchronic exposure to styrene on the extracellular and tissue levels of dopamine, serotonin and their metabolites in rat brain," Archives of Toxicology, vol. 80, no. 10, pp. 703$712,2006$.

[4] J. Kohn, S. Minotti, and H. Durham, "Assessment of the neurotoxicity of styrene, styrene oxide, and styrene glycol in primary cultures of motor and sensory neurons," Toxicology Letters, vol. 75, no. 1-3, pp. 29-37, 1995. 
[5] C. Moller, L. Odkvist, B. Larsby, R. Tham, T. Ledin, and L. Bergholtz, "Otoneurological findings in workers exposed to styrene," Scandinavian Journal of Work, Environment \& Health, vol. 16, no. 3, pp. 189-194, 1990.

[6] M. V. Vettori, D. Corradi, T. Coccini et al., "Styrene-induced changes in amacrine retinal cells: an experimental study in the rat," NeuroToxicology, vol. 21, no. 4, pp. 607-614, 2000.

[7] M. K. Viaene, W. Pauwels, H. Veulemans, H. A. Roels, and R. Masschelein, "Neurobehavioural changes and persistence of complaints in workers exposed to styrene in a polyester boat building plant: influence of exposure characteristics and microsomal epoxide hydrolase phenotype," Occupational and Environmental Medicine, vol. 58, no. 2, pp. 103-112, 2001.

[8] R. I. Kishi, Y. Katakura, T. Ikeda, B. Q. Chen, and H. Miyake, "Neurochemical effects in rats following gestational exposure to styrene," Toxicology Letters, vol. 63, no. 2, pp. 141-146, 1992.

[9] S. K. Chakrabarti, "Altered regulation of dopaminergic activity and impairment in motor function in rats after subchronic exposure to styrene," Pharmacology Biochemistry and Behavior, vol. 66, no. 3, pp. 523-532, 2000.

[10] E. Dare, R. Tofighi, L. Nutt et al., "Styrene 7,8-oxide induces mitochondrial damage and oxidative stress in neurons," Toxicology, vol. 201, no. 1-3, pp. 125-132, 2004.

[11] M. V. Vettori, A. Caglieri, M. Goldoni et al., "Analysis of oxidative stress in SK-N-MC neurons exposed to styrene-7,8oxide," Toxicology in Vitro, vol. 19, no. 1, pp. 11-20, 2005.

[12] J. A. Harvilchuck and G. P. Carlson, "Effect of multiple doses of styrene and R-styrene oxide on CC10, bax, and bcl-2 expression in isolated Clara cells of CD-1 mice," Toxicology, vol. 259, no. 3, pp. 149-152, 2009.

[13] IARC, "Styrene-7, 8-oxide," IARC Monographs on the Evaluation of Carcinogenic Risks to Humans, vol. 60, pp. 321-346, 1994.

[14] A. C. Johnson, T. C. Morata, A. C. Lindblad et al., "Audiological Wndings in workers exposed to styrene alone or in concert with noise," Noise \& Health, vol. 8, no. 30, pp. 45-57, 2006.

[15] G. Triebig, T. Bruckner, and A. Seeber, "Occupational styrene exposure and hearing loss: a cohort study with repeated measurements," International Archives of Occupational and Environmental Health, vol. 82, no. 4, pp. 463-480, 2009.

[16] G. D. Chen, C. Tanaka, and D. Henderson, "Relation between outer hair cell loss and hearing loss in rats exposed to styrene," Hearing Research, vol. 243, no. 1-2, pp. 28-34, 2008.

[17] R. Lataye, P. Campo, and G. Loquet, "Combined effects of noise and styrene exposure on hearing function in the rat," Hearing Research, vol. 139, no. 1-2, pp. 86-96, 2000.

[18] T. C. Morata, A. C. Johnson, P. Nylen et al., "CME article \#1: audiometric findings in workers exposed to low levels of styrene and noise," Journal of Occupational and Environmental Medicine, vol. 44, no. 9, pp. 806-814, 2002.

[19] M. sliwinska-Kowalska, E. Zamyslowska-Szmytke, W. Szymczak et al., "Ototoxic effects of occupational exposure to styrene and co-exposure to styrene and noise," Journal of Occupational and Environmental Medicine, vol. 45, no. 1, pp. 15-24, 2003.

[20] T. C. Morata and P. Campo, "Auditory function after single or combined exposure to styrene: a review," in Noise-Induced Hearing Loss: Basic Mechanisms, Prevention and Control, D. Henderson, Ed., pp. 293-304, Noise Research Network Publications, London, UK, 2001.

[21] G. D. Chen and D. Henderson, "Cochlear injuries induced by the combined exposure to noise and styrene," Hearing Research, vol. 254, no. 1-2, pp. 25-33, 2009.
[22] N. Cherry and D. Gautrin, "Neurotoxic effects of styrene: further evidence," British Journal of Industrial Medicine, vol. 47, no. 1, pp. 29-37, 1990.

[23] A. Fuente and B. McPherson, "Organic solvents and hearing loss: the challenge for audiology," International Journal of Audiology, vol. 45, no. 7, pp. 367-381, 2006.

[24] K. V. Gopal, "Audiological findings in individuals exposed to organic solvents: case studies," Noise and Health, vol. 10, no. 40, pp. 74-82, 2008.

[25] E. Zamyslowska-Szmytke, A. Fuente, E. Niebudek-Bogusz, and M. Sliwinska-Kowalska, "Temporal processing disorder associated with styrene exposure," Audiology and Neurotology, vol. 14, no. 5, pp. 296-302, 2009.

[26] EU, "Directive 2003/10/EC of the European parliament and of the council of 6 February 2003 on the minimum health and safety requirements regarding the exposure of workers to the risks arising from physical agents (noise)," Official Journal L, vol. 42, pp. 38-44, 2003.

[27] National Institute for Occupational Safety and Health, Hearing Loss Research at NIOSH: Reviews of Research Programs of the NIOSH, National Academies Press, Washington, DC, USA, 2006.

[28] P. Hoet and D. Lison, "Ototoxicity of toluene and styrene: state of current knowledge," Critical Reviews in Toxicology, vol. 38, no. 2, pp. 127-170, 2008.

[29] K. V. Gopal and G. W. Gross, "Auditory cortical neurons in vitro: cell culture and multichannel extracellular recording," Acta Oto-Laryngologica, vol. 116, no. 5, pp. 690-696, 1996.

[30] K. V. Gopal and G. W. Gross, "Unique responses of auditory cortex networks in vitro to low concentrations of quinine," Hearing Research, vol. 192, no. 1-2, pp. 10-22, 2004.

[31] K. V. Gopal, B. R. Miller, and G. W. Gross, "Acute and subchronic functional neurotoxicity of methylphenidate on neural networks in vitro," Journal of Neural Transmission, vol. 114, no. 11, pp. 1365-1375, 2007.

[32] G. W. Gross, "Internal dynamics of randomized mammalian neuronal networks in culture," in Enabling Technologies for Cultured Neural Networks, D. A. Stenger and T. M. McKenna, Eds., pp. 277-317, Academic Press, New York, NY, USA, 1994.

[33] G. W. Gross and J. M. Kowalski, "Experimental and theoretical analysis of random nerve cell network dynamics," in Neural Netwoks: Concepts, P. Antognetti and V. Milutnovec, Eds., pp. 47-100, Prentice Hall, Englewood, NJ, USA, 1991.

[34] G. W. Gross, W. Y. Wen, and J. W. Lin, “Transparent indiumtin oxide electrode patterns for extracellular, multisite recording in neuronal cultures," Journal of Neuroscience Methods, vol. 15, no. 3, pp. 243-252, 1985.

[35] S. O. Rijal and G. W. Gross, "Dissociation constants for $\mathrm{GABA}_{\mathrm{A}}$ receptor antagonists determined with neuronal networks on microelectrode arrays," Journal of Neuroscience Methods, vol. 173, no. 2, pp. 183-192, 2008.

[36] G. W. Gross, "Multielectrode arrays," Scholarpedia, vol. 6, no. 3, Article ID 5749, 2011.

[37] I. Morioka, M. Kuroda, K. Miyashita, and S. Takeda, "Evaluation of organic solvent ototoxicity by the upper limit of hearing," Archives of Environmental Health, vol. 54, no. 5, pp. 341-346, 1999. 


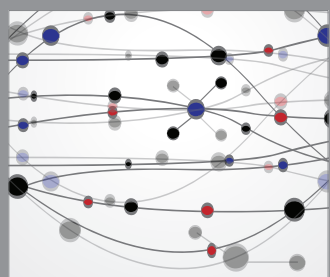

The Scientific World Journal
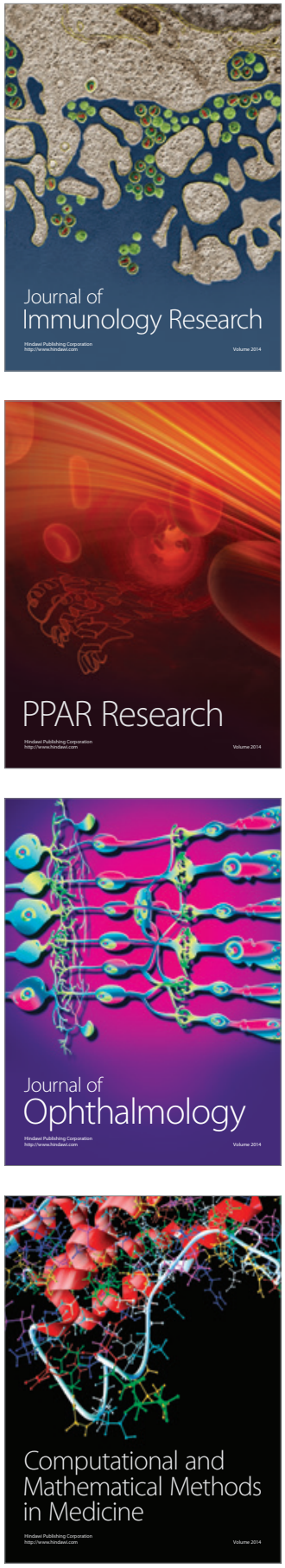

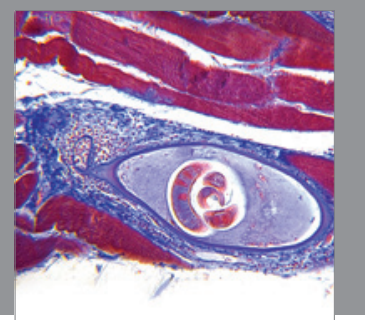

Gastroenterology

Research and Practice
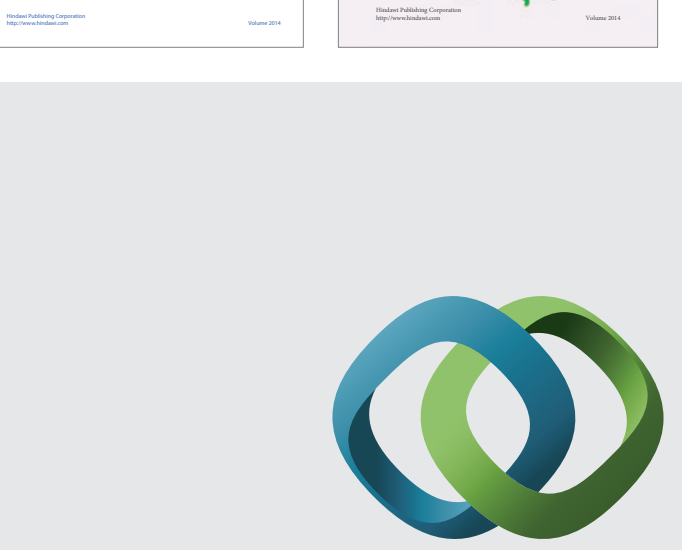

\section{Hindawi}

Submit your manuscripts at

http://www.hindawi.com
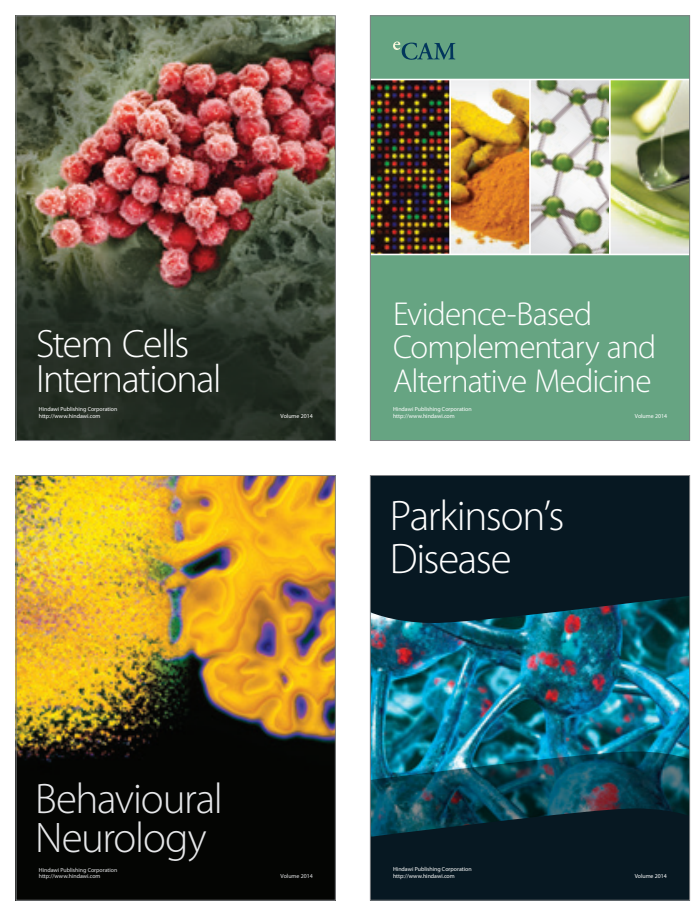

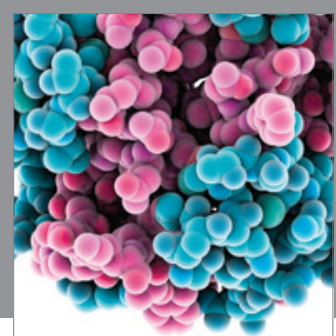

Journal of
Diabetes Research

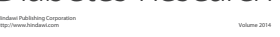

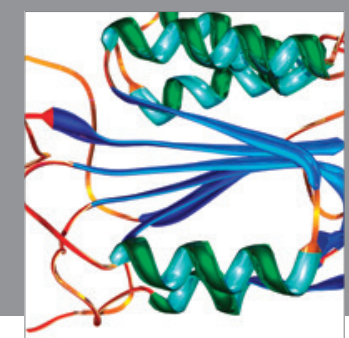

Disease Markers
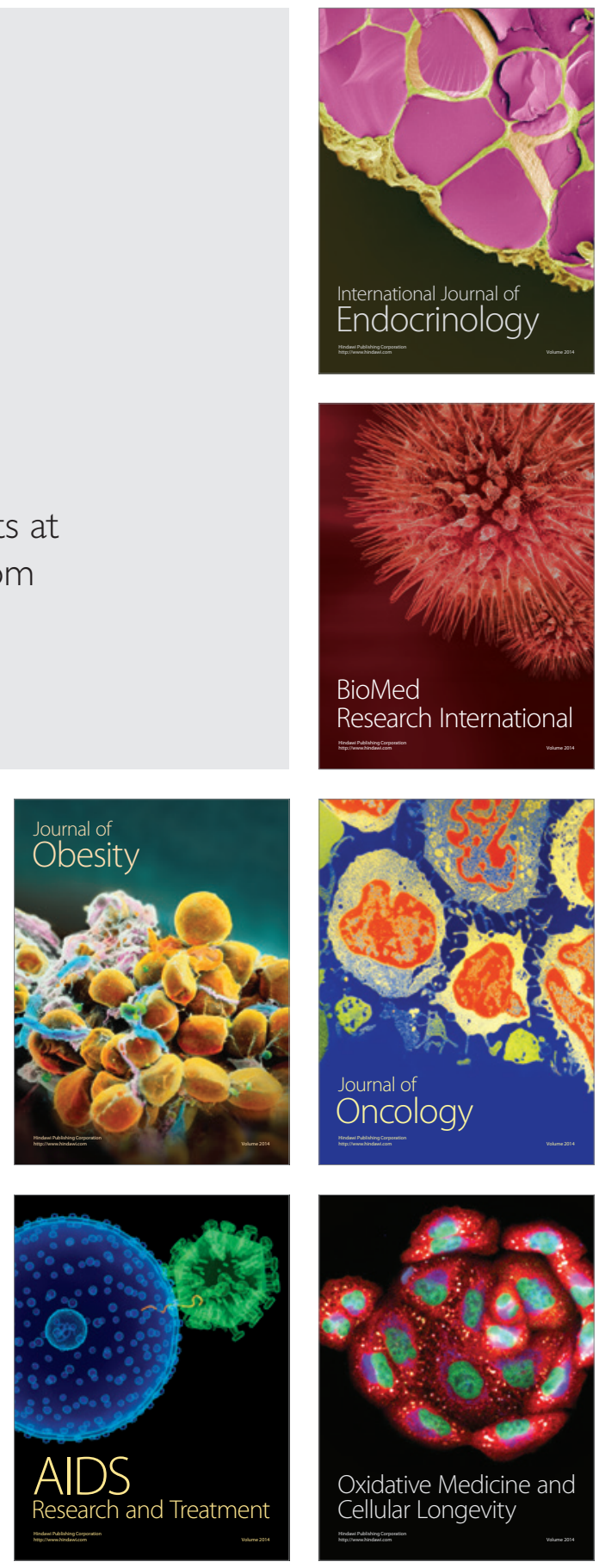